\title{
MAXimum ANd Minimum Power ADAPTATION ANALYSIS FOR TRANSMISSION OF DIFFERENT IMAGE FORMATS USING UNILEVEL HAAR WAVELET
}

\author{
M Padmaja ${ }^{1}$, Dr. P. Satyanarayana ${ }^{2}$, K. Prasuna ${ }^{3}$ \\ ${ }^{1}$ Assoc. Prof., ECE Dept., VR Siddhartha Engg. College, Vijayawada (A.P.), India \\ ${ }^{2}$ Prof., ECE, Sri Venkateswara University College of Engg. Tirupati (A.P.), India \\ ${ }^{3}$ Asst. Prof., ECE Department, Vijaya Institute of Technology for Women, A.P., India \\ ${ }^{1}$ padmaja19m@gmail.com, ${ }^{3}$ prasuna.k@rediffmail.com
}

\begin{abstract}
This paper addresses power allocation methods for multimedia signals over wireless channels. The aim of this paper is to minimize total power allocated for image compression and transmission, while the power for each bit is kept at a predetermined value. Maximum and minimum power control algorithms are proposed. In this work, an approach for minimizing the total power allocated to a multimedia like image due to source compression and transmission subject to a fixed bit source distortion using different parameters are analysed. Simulations are performed using haar wavelet over AWGN channel. Structural Content (SC), Average Difference, Normalized Absolute Error (NAE), etc are analysed. Maximum Power Adaptation Algorithm shows better performance than Conventional Power Adaptation Algorithm.
\end{abstract}

Keywords - PSNR, MSE, SC, NAE, Correlation, Image formats

\section{INTRODUCTION}

One of the most important and challenging goal of current and future communication is transmission of high quality images from source to destination quickly with least error where limitation of bandwidth is a prime problem. By the advent of multimedia communications, the multimedia transmission of multimedia over wireless links is considered as one of the major applications of future communication systems. However, such systems require the use of relatively high power adaptation compared to other applications. With such requirement, it is very challenging to provide acceptable quality of services as measured by the Root Mean Square Error (RMSE) due to the limitations imposed by the wireless communication channels such as fading and multipath propagation.

With the increasing complexity of these communication systems comes increasing complexity in the type of content being transmitted and received. The early content of plain speech/audio and basic black and white images used in early radio and television has developed into high definition audio and video streams; and with the introduction of computers into the mix even more complex content needs to be considered from images, video and audio to medicaland financial data. Techniques are continuously being developed to maximise data throughput and efficiency in these wireless communication systems while endeavouring to keep data loss and error to a minimum.Power control has been an effective approach to mitigating the effect of fading channels in the quality of signal transmission over wireless channels[1-2]. 
Signal \& Image Processing : An International Journal (SIPIJ) Vol.3, No.4, August 2012

\section{PROBLEM FORMULATION}

Efficient use of the multimedia power is one of the major challenges in information devices. Some of the key technologies that affect the power in this respect are source signal compression, channel error control coding, and radio transmission. Depending on the Allocation, power control algorithms can be categorized as either centralized or distributed. An optimum centralized power control algorithm which can achieve the minimum outage probability was studied in [3]. It is assumed that all the active link gains are available and remain constant during execution of the algorithm. This assumption, of course, is not realistic because of the high computational complexity required for the algorithm [4] [5]. In the previous algorithms of power allocation methods only local information is used to adjust transmitting power. In this paper, a power adaptation algorithm which does not need the normalization procedure is proposed [6, [7], [8].

\section{OPTIMIZATION Using PoWer Allocation Methods}

When there are $\mathrm{N}$ number of images and $\mathrm{M}$ number of bits in a multimedia system, then the powers transmitted by the bits beP $=\left[\mathrm{P}_{1}, \mathrm{P}_{2}, \ldots \ldots \ldots \ldots \ldots \mathrm{P}_{\mathrm{M}}\right]$ and the respective RMSEs at the bits be RMSE $=\left[\mathrm{RMSE}_{1}, \mathrm{RMSE}_{2}, \ldots \ldots \ldots . \mathrm{RMSE}_{\mathrm{M}}\right]$. Let $R M S E_{T}$ be the target RMSE.

A Communication system requires $2^{\mathrm{M}}$ different samples to be transmitted for $\mathrm{M}$ bits per sample.

The mean square error (MSE) is given by

$$
\operatorname{MSE}=\sum_{j=0}^{2^{M}-1}\left(x_{j}-x_{j}\right)^{2} P\left(x_{j}\right)
$$

Where $x \mathrm{j}$ is the estimate of the $\mathrm{j}^{\text {th }}$ sample reconstructed after detection of the $\mathrm{M}$ bits and $\mathrm{P}\left(\mathrm{x}_{\mathrm{j}}\right)$ is the a priori probability that the jth sample is transmitted. In general, $2^{\mathrm{M}}-1$ combinations of $\mathrm{M}$ bits will be received. [17]. The probability that ith sample with a decimal value of (i) is reconstructed is given by

$$
P_{i}=\prod_{k=0}^{M-1}\left[p_{k} \vartheta(k)+\left(1-p_{k}\right) \widetilde{\vartheta(k)}\right]
$$

Where $p_{k}$ is the probability that the kth bit is in error. $\vartheta(k)$ is equal to zero if the indices of $i$ and $\mathrm{k}$ are same and the value will be equal to 1 if the indices are different. The notation $\overline{\vartheta(\mathrm{k})}$ ] represents the binary inversion of $\vartheta(\mathrm{k})$.

The MSE for the above case is calculated as

$$
\operatorname{MSE}=\frac{1}{\sqrt{2^{\mathrm{M}}-1}} \sum_{\mathrm{k}=0}^{\mathrm{M}-1} \mathrm{P}_{\mathrm{i}}
$$

The Root Mean Square Error (RMSE) is obtained by taking the square root of (3)[15-18] .Note that the probability of the kth bit to be in error for the AWGN case is given by

$$
P E_{k}=Q\left(\sqrt{2 \frac{E_{b}}{N_{o}}}(k)\right)
$$

\section{Minimum Power Adaptation Algorithm}

This algorithm is developed to minimize the interference experienced by a channel such that heterogeneous BER requirements of multimedia traffic are satisfied. Further, from analysis of the maximum capacity of a bit stream, it is concluded that both transmission rate and BER are necessary to reach a maximum capacity. A new scheme is proposed to serve in order to maximize 
the capacity of such an interference-sensitive system, the power levels allocated to code channels need to be minimized.[9-12]

\section{ALGORITHM:}

1. Initialize the power distribution vector to all ones

2. For 1 to No. of iterations and No. of bits, calculate the MSE using (5) and (6) and keep changing the energy of the two bits until you find the minimum value of MSE and update the power of all the bits using

$$
\mathrm{P}_{\mathrm{i}}^{\mathrm{n}+1}=\mathrm{RMSE}_{\mathrm{i}}^{\mathrm{n}} \times \mathrm{P}_{\mathrm{i}}^{\mathrm{n}}
$$

Where

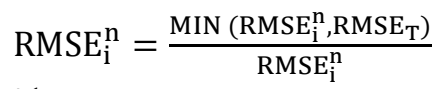

$\mathrm{P}_{\mathrm{i}}^{\mathrm{n}+1}=$ Power allocated in the $\mathrm{n}+1$ state

$\mathrm{P}_{\mathrm{i}}^{\mathrm{n}}=$ Power allocated in the $\mathrm{n}$ state

RMSE $_{\mathrm{i}}^{\mathrm{n}}=$ Root mean square error of ith bit in $\mathrm{n}^{\text {th }}$ iteration

$\mathrm{RMSE}_{\mathrm{T}}=$ Target Root Mean Square error

3. Define two bits, $\mathrm{R}$ is recipient power and $\mathrm{C}$ is contributing power and assumes the power step size to $\Delta \mathrm{P}$.

4. Calculate the minimum power of each bit.Repeat the same procedure (2) and (3) above but with the contributor bit $\mathrm{C}$ is incremented by one until all least significant bits are used.

5. Calculate the Minimum MSE.

\section{Maximum Power Adaptation Algorithm}

In these systems, the MSE level is satisfied at each bit. Once the bit allocation is carried out, the power control takes a role of controlling the error caused by bits. On one hand, this algorithm must be reduced to minimize the interference at other bits, and, on the other hand, it must be sufficient for data communication[23-24].

\section{ALGORITHM:}

1. Initialize the power distribution vector to all ones

2. For 1 to No. of iterations and No. of bits, calculate the MSE using (5) and (7) and keep changing the energy of the two bits until you find the minimum value of MSE and update the power of all the bits using

Where

$$
\begin{aligned}
& P_{i}^{n+1}=\operatorname{RMSE}_{i}^{n} x P_{i}^{n} \\
& \quad \operatorname{RMSE}_{i}^{n}=\frac{\operatorname{MAX}\left(\operatorname{RMSE}_{i}^{n}, \operatorname{RMSE}_{T}\right)}{\operatorname{RMSE}_{\mathrm{i}}^{\mathrm{n}}}
\end{aligned}
$$

$\mathrm{P}_{\mathrm{i}}^{\mathrm{n}+1}=$ Power allocated in the $\mathrm{n}+1$ state

$\mathrm{P}_{\mathrm{i}}^{\mathrm{n}}=$ Power allocated in the $\mathrm{n}$ state

RMSE $_{\mathrm{i}}^{\mathrm{n}}=$ Root mean square error of ith bit in $\mathrm{n}^{\text {th }}$ iteration RMSE $_{\mathrm{T}}=$ Target Root Mean Square error

3. Define two bits, $\mathrm{R}$ is recipient power and $\mathrm{C}$ is contributing power and assumes the power step size to $\Delta \mathrm{P}$. 
Signal \& Image Processing : An International Journal (SIPIJ) Vol.3, No.4, August 2012

4. Calculate the maximum power of each bit. Repeat the same procedure (2) and (3) above but with the contributor bit $\mathrm{C}$ is incremented by one until all least significant bits are used.

5. Calculate the Maximum MSE.

\section{WA VELETS AND IMAGE FORMATS}

Wavelets are mathematical functions that cut up data into different frequency components, and then study each component with a resolution matched to its scale [17]. The wavelet transform has the ability to decorrelate an image both in space and frequency there by distributing energy compactly into a few low frequency and a high frequency coefficients. The efficiency of a wavelet based image compression scheme depends both on the wavelet filters chosen as well as on the coefficient quantization scheme. An image can be decomposed into subbands. These sub bands are decomposed into LL,LH,HL and HH bands. The LL band is transmitted along the channel by allocating power allocation and one level of decomposition was taken into consideration. The four bands are transmitted over wireless channel and the coefficients are reconstructed using inverse transform. The approximation coefficients are reconstructed using inverse discrete transform process and various parameters are studied in the proposed and conventional methods for one level of sub band decomposition.

There are different image formats available for different applications. In this paper, four types of formats., PNG, BMP, TIF and GIF are considered.Each format undergoes one different application in real-time.

\section{NUMERICAL RESULTS AND CONCLUSIONS}

Objective and Subjective quality parameter values are obtained for Equal Power Adaptation Algorithm, Minimum Power Adaptation Algorithm and Maximum Power Adaptation Algorithms. The improvement in performance is obtained by the Minimum Power allocation Algorithm which affects the Maximum Power Adaptation Algorithm performance in comparison as shown in Table I, Table II, Table III.

Fig.1, Fig. 2 and Fig. 3 shows the plots of different parameters such as Mean Square Error, Peak Signal To Noise Ratio (PSNR), Average Difference (AD), Normalized Average Error (NAE), etc for Maximum, minimum and Conventional Equal power Adaptation methods. Both Minimum Adaptation Algorithm and Maximum Power Adaptation Algorithms show better performance in image transmission using unilevel of haar wavelet compared with Conventional Power Adaptation as shown in Fig.4, Fig.5, Fig.6.

In all the three methods, Maximum Power Adaptation algorithm (MAPCAA) shows better performance with less error with all remaining formats. Each and every image format has its own characteristic in quality performance. 
Signal \& Image Processing : An International Journal (SIPIJ) Vol.3, No.4, August 2012

\begin{tabular}{|c|c|c|c|c|}
\hline $\begin{array}{c}\text { Power Allocation } \\
\text { Method }\end{array}$ & \multicolumn{4}{|c|}{ MPAA } \\
\hline Image Format & Barbara.png & Lena.bmp & Cameraman.tif & Lena1.gif \\
\hline MSE & $2.76 \mathrm{E}+03$ & $3.66 \mathrm{E}+03$ & $3.78 \mathrm{E}+03$ & $1.66 \mathrm{E}+03$ \\
\hline PSNR & $1.37 \mathrm{E}+01$ & $1.25 \mathrm{E}+01$ & $1.24 \mathrm{E}+01$ & $1.59 \mathrm{E}+01$ \\
\hline MNCorr. & 0.9117 & $1.03 \mathrm{E}+00$ & 0.8526 & 0.952 \\
\hline AD & -10.19 & $-3.17 \mathrm{E}+01$ & -8.8678 & -6.8534 \\
\hline SC & 1.0097 & $7.30 \mathrm{E}-01$ & 1.0921 & 0.9941 \\
\hline MD & 123 & $1.09 \mathrm{E}+02$ & 123 & 86 \\
\hline NAE & 0.373 & $5.23 \mathrm{E}-01$ & 0.4124 & 0.277 \\
\hline Elapsed time (sec) & 7.621745 & 7.672785 & 7.673764 & 9.840020 \\
\hline STD & 10.5236 & $1.06 \mathrm{E}+01$ & 13.6214 & 8.8657 \\
\hline SFM & 0.3935 & $3.77 \mathrm{E}-01$ & 0.25 & 0.3045 \\
\hline En & 5.06917 & $4.84 \mathrm{E}+00$ & 4.5857 & 4.386662 \\
\hline Correlation & 0.1969 & $1.98 \mathrm{E}-01$ & 0.2165 & 0.2097 \\
\hline
\end{tabular}

Table. I Table showing different parameters using Minimum Power Adaptation Algorithm for different image formats

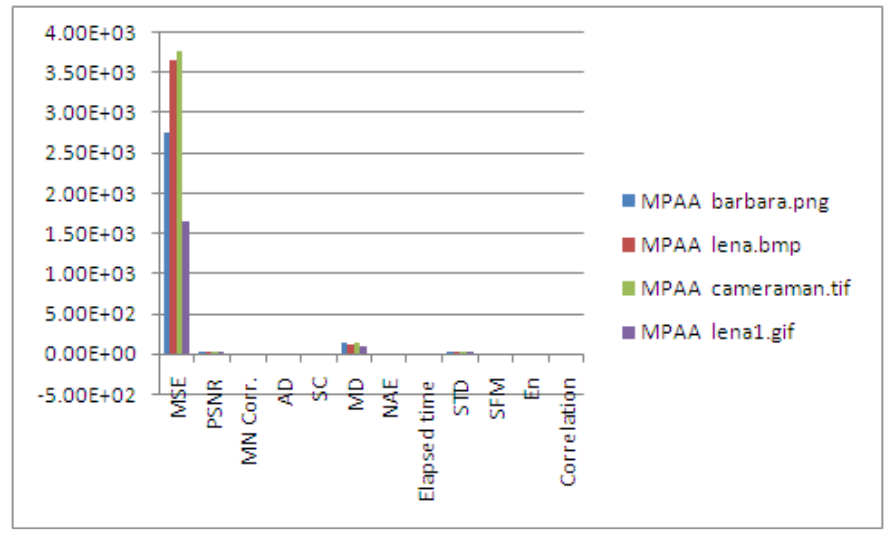

Fig.1 Plot showing different parameters using Minimum Power Adaptation Algorithm for different image formats

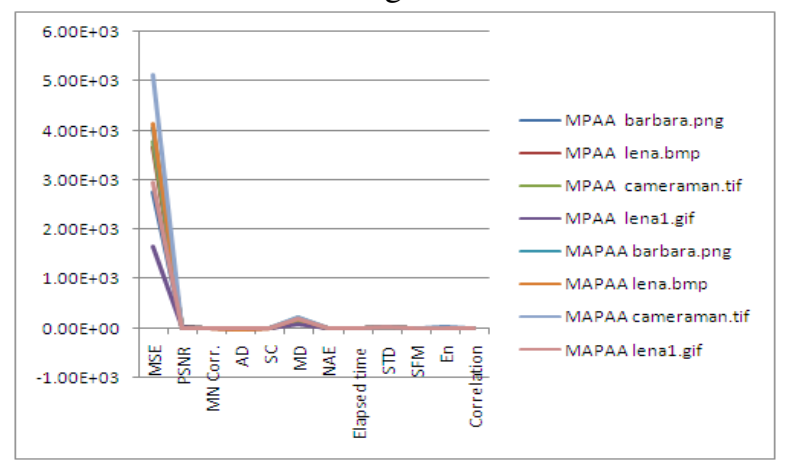

Fig.2 Plot showing different parameters using Minimum and Maximum Power Adaptation Algorithm for different image formats 
Signal \& Image Processing : An International Journal (SIPIJ) Vol.3, No.4, August 2012

\begin{tabular}{|c|c|c|c|c|}
\hline $\begin{array}{c}\text { Power Allocation } \\
\text { Method }\end{array}$ & \multicolumn{4}{|c|}{ MAPAA } \\
\hline Image Format & Barbara.png & Lena.bmp & Cameraman.tif & Lena1.gif \\
\hline MSE & $4.06 \mathrm{E}+03$ & $4.14 \mathrm{E}+03$ & $5.13 \mathrm{E}+03$ & $2.95 \mathrm{E}+03$ \\
\hline PSNR & $1.20 \mathrm{E}+01$ & $1.20 \mathrm{E}+01$ & $1.10 \mathrm{E}+01$ & $1.34 \mathrm{E}+01$ \\
\hline MNCorr. & 0.7895 & 0.8979 & 0.7394 & 0.8283 \\
\hline AD & 6.6688 & -14.9932 & 7.8111 & 9.8273 \\
\hline SC & 1.2134 & 0.8754 & 1.3087 & 1.1931 \\
\hline MD & 224 & 214 & 227 & 202 \\
\hline NAE & 0.4368 & 0.55 & 0.4824 & 0.35 \\
\hline Elapsed time (sec) & 7.798703 & 7.663775 & 7.606087 & 7.639787 \\
\hline STD & seconds & seconds & seconds & seconds \\
\hline SFM & 37.1228 & 36.9908 & 37.9231 & 36.4632 \\
\hline En & 0.3916 & 0.3767 & 0.2499 & 0.3038 \\
\hline Correlation & 6.052191 & 5.912548 & 5.735599 & 5.604073 \\
\hline & 0.034 & 0.0552 & 0.0538 & 0.0534 \\
\hline
\end{tabular}

Table. II Table showing different parameters using Maximum Power Adaptation Algorithm for different image formats

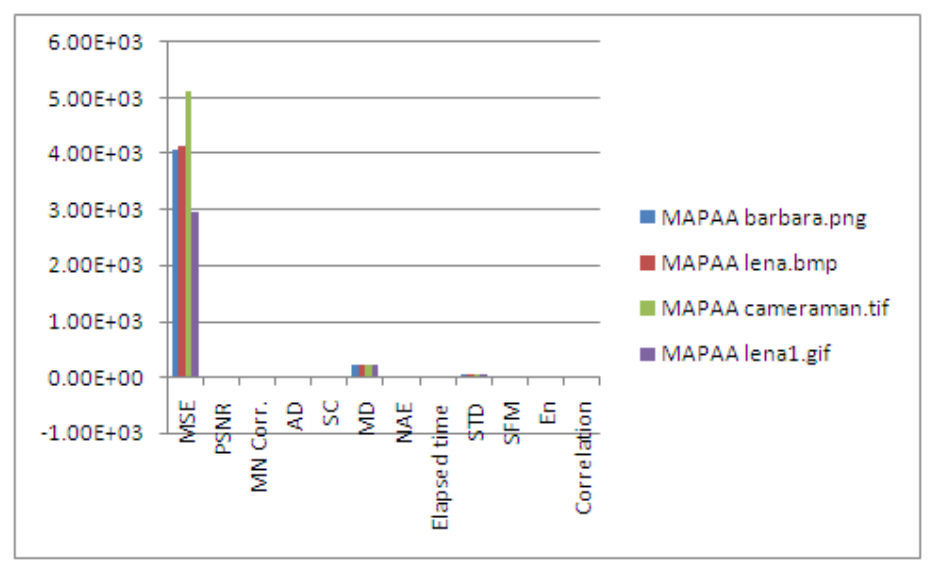

Fig.3 Plot showing different parameters using Maximum Power Adaptation Algorithm for different image formats 
Signal \& Image Processing : An International Journal (SIPIJ) Vol.3, No.4, August 2012

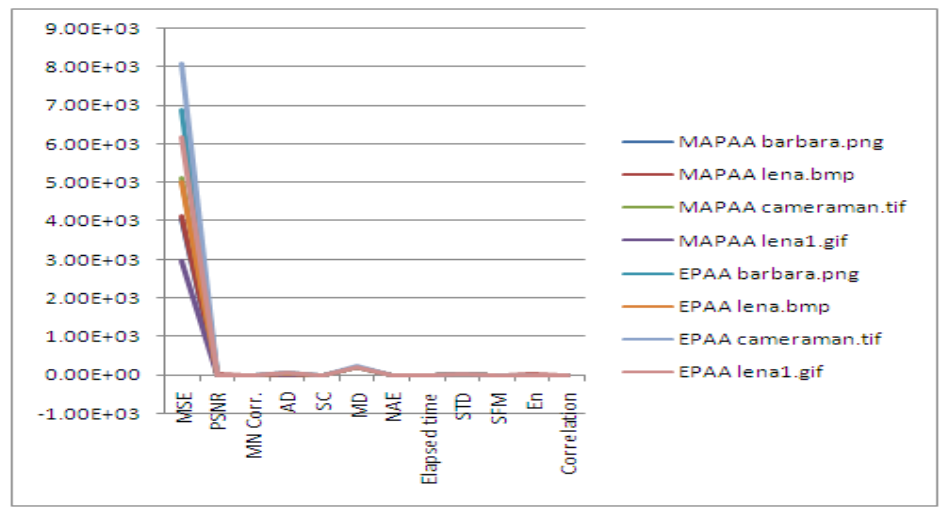

Fig.4 Plot showing different parameters using Maximum and Equal Power Adaptation Algorithm for different image formats

Table. III Table showing different parameters using Equal Power Adaptation Algorithm for different image formats

\begin{tabular}{|c|c|c|c|c|}
\hline $\begin{array}{c}\text { Power Allocation } \\
\text { Method }\end{array}$ & \multicolumn{4}{|c|}{ EPAA } \\
\hline Image Format & Barbara.png & Lena.bmp & Cameraman.tif & Lenal.gif \\
\hline MSE & 6890 & 5020 & $8.08 \mathrm{E}+03$ & $6.18 \mathrm{E}+03$ \\
\hline PSNR & 9.75 & 11.12 & $9.06 \mathrm{E}+00$ & 10.2194 \\
\hline MNCorr. & 0.46 & 0.524 & 0.4313 & 0.4804 \\
\hline AD & 53.5 & 31.64 & 54.532 & 56.4457 \\
\hline SC & 2.99 & 2.139 & 3.209 & 2.9304 \\
\hline MD & 223 & 219 & 237 & 209 \\
\hline NAE & 0.58 & 0.592 & 0.6555 & 0.5408 \\
\hline Elapsed time (sec) & 21.469618 & 22.067712 & 23.430954 & 22.361518 \\
\hline STD & 38.3 & 38.29 & 38.5099 & 37.6496 \\
\hline SFM & 0.39 & 0.37 & 0.2422 & 0.2978 \\
\hline En & 7.08 & 7.085 & 7.098955 & 7.06968 \\
\hline Correlation & 0.05 & 0.053 & $5.58 \mathrm{E}-02$ & 0.0341 \\
\hline
\end{tabular}

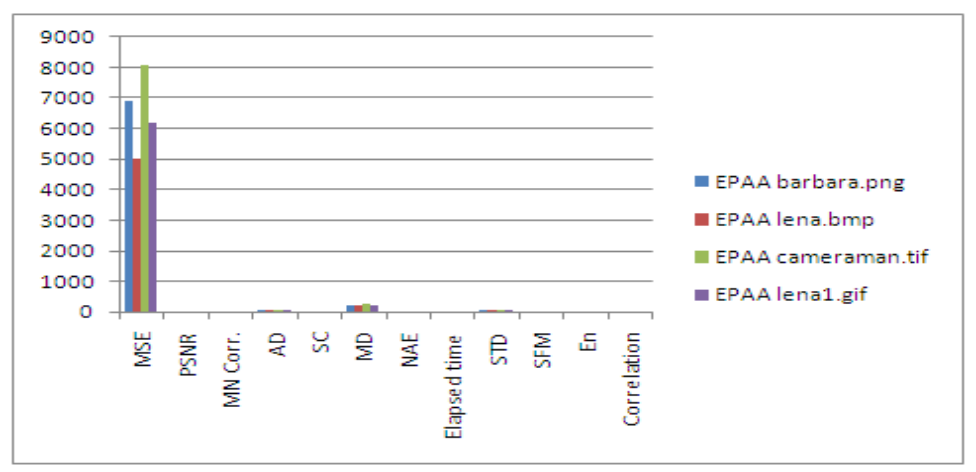

Fig.5 Plot showing different parameters using Equal Power Adaptation Algorithm for different image formats 


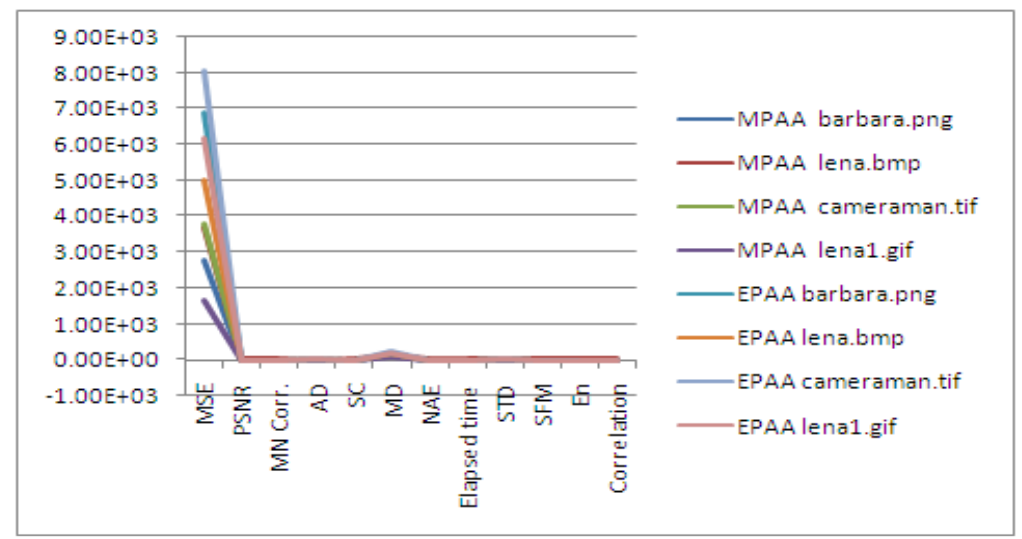

Fig.6 Plot showing different parameters using Minimum and Equal Power Adaptation Algorithm for different image formats

\section{REFERENCES}

[1] R. Zhang, S. L. Regunathan, and K. Rose, "Video coding with optimal inter/intra-mode switching for packet loss resilience,” IEEE J. Select. Areas Commun., vol. 18, pp. 966-976, June 2000.

[2] K. Stuhlmüller, N. Färber, M. Link, and B. Girod, "Analysis of video transmission over lossy channels,” IEEE J. Select. Areas Commun. , vol. 18, pp. 1012-1032, June 2000.

[3] T. H. Meng, A. C. Hung, E. K. Tsern, and B. M. Gordon, "Low-power signal processing system design for wireless applications,” IEEE Pers. Commun. , pp. 20-31, June 1998.

[4] S. Appadwedula, M. Goel, N. R. Shanbhag, D. L. Jones, and K. Ram-chandran, “Total system energy minimization for wireless image trans-mission,” J. VLSI Signal Processing Syst., vol. 27, no. 1/2, pp. 99-117,Feb. 2001.

[5] Q. Zhang, Z. Ji, W. Zhu, and Y. Q. Zhang, "Power-minimized bit allo-cation for video communication over wireless channels," IEEE Trans. Circuits Syst. Video Technol., vol. 6, pp. 398410, June 2002.

[6] Technical specification group radio access network:physical layer -general specification, 3GPP, Release 6,December 2003, www.3gpp.org.

[7] J. Proakis, Digital Communications, 4th ed., McGraw-Hill,2001.Talukder, K.H. and Harada, K., A Scheme of Wavelet Based Compression of 2D Image, Proc. IMECS, Hong Kong, pp. 531-536, June 2006.

[8] Ahmed, N., Natarajan, T., and Rao, K. R., Discrete Cosine Transform, IEEE Trans. Computers, vol. C-23, Jan. 1974, pp. 90-93.

[9] Pennebaker, W. B. and Mitchell, J. L. , Still Image Data Compression Standards, Van Nostrand Reinhold, 1993. Press, 1990.

[10] Wallace, G. K., The Still Picture Compression Standard , Comm. ACM, vol. 34, no. 4, April 1991, pp. 30-44.

[11] Colm Mulcahy, Image compression using the Haar Wavelet transforms , Internal Report.

[12] Wireless Communications by Andrea Goldsmith 


\section{Authors}

Ms M Padmaja is currently working as Associate Professor in the Department of ECE, VR Siddhartha Engineering College, Vijayawada. She is working towards her Ph.D at Sri Venkateswara University College of Engineering, Tirupati, under the guidance of Dr P Sathyanarayana. She has 16years of experience in teaching various courses at B.Tech and M.Tech. Her research areas are Signal Processing and Communications. She has more than 12 research papers to her credit. She is Fellow of IETE, Life member of IE (I), ISTE, SEMCEI, and BMESI.

Dr P Sathyanarayana received the B. E., M. E., and Ph. D. degrees from Sri Venkateswara University, Tirupati, A. P., India, in 1976, 1978 and 1987 respectively all in Electronics and Communication Engineering. He Joined the Department of Electrical and Electronics Engineering, Sri Venkateswara University College of Engineering, Tirupati, in July 1979 as an assistant Professor and became Professor in July 1999. His research interests are in the field of one and two dimensional Signal Processing particularly in Image/ Video processing and Communication.He was with the Dept. of Electrical and Computer Engg., Concordia University Montreal, Canada from 1988 to 1990 as Post Doctoral Fellow,

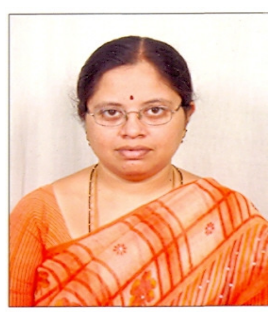
and he was with School of Aerospace Engg., University Sains Malaysia, Penang, Malaysia as a visiting faculty from 2004 to 2007.

Ms K Prasuna obtained M.Tech in Communications and Signal Processing from Acharya Nagarjuna University in 2009. She has more than 5 years of teaching experience. Presently she is working as Assistant Professor in ECE department in Vijaya Institute of Technology for Women, Vijayawada. Her areas of interest are Digital Signal Processing, Wireless Communications, Image Processing and Wireless Networks.

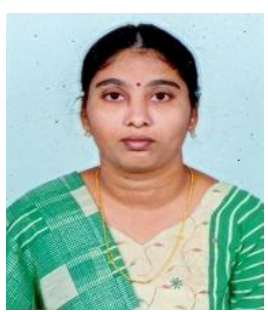

